UTAS-PHYS-96-08

\title{
On boson algebras as Hopf algebras
}

\author{
I Tsohantjis ${ }^{\dagger}$ A Paolucci* and P D Jarvis ${ }^{\dagger}$ \\ ${ }^{\dagger}$ Department of Physics, University of Tasmania \\ GPO Box 252C Hobart, Australia 7001 \\ * School of Mathematics, University of Leeds, \\ Leeds, LS2 9JT UK
}

\begin{abstract}
Certain types of generalized undeformed and deformed boson algebras which admit a Hopf algebra structure are introduced, together with their Fock-type representations and their corresponding $R$-matrices. It is also shown that a class of generalized Heisenberg algebras including those underlying physical models such as that of Calogero-Sutherland, is isomorphic with one of the types of boson algebra proposed, and can be formulated as a Hopf algebra.
\end{abstract}

Physics and Astronomy Classifications. 02.10. 


\section{Introduction.}

Deformations of the boson algebra have been recently the subject of extensive research partly because of their significance in quantum groups (see for example [1]-[5]) and supergroups [6]. Chronologically first comes the Arik-Coon $q$-deformation of the Heisenberg algebra [7]:

$$
a a^{\dagger}-q a^{\dagger} a=I,
$$

followed by the Macfarlane-Biedenharn [8, 9], and Sun and Fu [10] $q$-deformed bosons

$$
a a^{\dagger}-q^{ \pm} a^{\dagger} a=q^{\mp N},
$$

the Chakrabarti-Jagannathan two-parameter model [11

$$
a a^{\dagger}-p a^{\dagger} a=q^{-N}
$$

and the Calogero-Vasiliev model [12]

$$
\left[a, a^{\dagger}\right]=I+2 \nu K, \quad K^{2}=I
$$

which coupled with (2) as

$$
a a^{\dagger}-q a^{\dagger} a=q^{-N}(I+2 \nu K)
$$

was studied in [13], while its $q$-deformation by Macfarlane [14] is

$$
a a^{\dagger}-q^{ \pm(I+2 \nu K)} a^{\dagger} a=[I+2 \nu K]_{q} q^{\mp(N+\nu-\nu K)}, \quad K=(-1)^{N},
$$

where as usual $[x]_{q}=\left(q^{x}-q^{-x}\right) /\left(q-q^{-1}\right)$. In addition with the Katriel-Quesne minimally deformed oscillators [28] which provides an attempt to unify existing deformed oscillators, generalizations and applications of the above models to mathematics and physics have been of increasing interest, and their consistency, interrelation and representations have been well analyzed [15], [16] [17]-27].

Generalizations of the usual Heisenberg algebra that have appeared in [12] have been implemented [29], [30], in describing relativistic fields with arbitrary fractional spin (anyon), "bosonizing" supersymmetric quantum mechanics and pointing out the relation of it with integrable quantum mechanical models [31] such as the Calogero-Sutherland model [32], [33].

On the other hand, the recent investigation [34]-[38] of simplest $q$-deformations of the Heisenberg algebra has also been shown to play a key part in obtaining and classifying representations of deformed bosons algebras [39].

The relation of a possible Hopf algebra structure consistent both with an appropriate definition of a boson algebra and its deformation have also been addressed [23], [40]- 43]. In particular in [41], [42], [43] a certain definition of deformed boson algebra was investigated having a Hopf algebra structure, while in [44] the $R$-matrix obtained was corrected and generalized using the quantum double construction. The results of [41] were partly generalized in [23].

The aim of this paper is to investigate certain generalizations of undeformed and deformed boson algebras possessing a Hopf algebra structure, which in [45] will be used to establish an algebraic relation with already known boson algebras (undeformed and deformed). In section two of this paper we introduce general notions on quasitriangular Hopf algebras. In section three we introduce and analyze the properties of undeformed generalized boson algebras $B_{\alpha, \beta}$ and $\bar{B}_{\sigma, \tau}$ which admit a Hopf algebra structure, while in section four we give a $q$-deformation 
of the previous algebras, prove that they also admit a Hopf structure and present an $R$-matrix for each one of them. We further analyze in section five a more general form of the 'deformed' Heisenberg algebra $H_{\nu}$ of [12], showing that under certain conditions it admits a Hopf algebra structure and demonstrate its connection with the undeformed boson algebra $B_{\alpha, \beta}$ defined in the third section. Finally we end with certain comments on possible physical and mathematical applications and consequences of our approach.

\section{Generalities on quasitriangular Hopf algebras.}

Consider a unital associative algebra, over a field $F$, with multiplication $m: A \otimes A \rightarrow A$ (i.e. $m(a \otimes b)=a b, \forall a$ and $\forall b \in A$ ) and unit $u: F \rightarrow A$ (i.e. $u(1)=I$, the identity on $A$ ) endowed with a Hopf algebra structure, that is, having a coproduct $\Delta: A \rightarrow A \otimes A$, a counit $\varepsilon: A \rightarrow F$ (which is a homomorphism) and an antipode $S: A \rightarrow A$ (which is an antihomomorhism i.e. $S(a b)=S(b) S(a)$, and we shall assume that it has an inverse $S^{-1}$ ) subject to the following consistency condition:

$$
\begin{gathered}
(i d \otimes \Delta) \Delta(a)=(\Delta \otimes i d) \Delta(a) \\
(i d \otimes \varepsilon) \Delta(a)=(\varepsilon \otimes i d) \Delta(a)=a \\
m(i d \otimes S) \Delta(a)=m(S \otimes i d) \Delta(a)=\varepsilon(a) I \quad \forall a \in A .
\end{gathered}
$$

Following Sweedler 47 we write

$$
\begin{gathered}
\Delta(a)=\sum_{(a)} a^{(1)} \otimes a^{(2)} \\
\Delta_{n}(a)=\left(\Delta \otimes I^{\otimes(n-1)}\right) \Delta_{n-1}(a)=\sum_{(a)} a^{(1)} \otimes a^{(2)} \ldots \otimes a^{(n+1)}
\end{gathered}
$$

Let $T$ be the twist map on $A \otimes A$ defined by $T(a \otimes b)=b \otimes a$. Then there also exists an opposite Hopf algebra structure on $A$ with coproduct $T \Delta \doteq \Delta^{T}$, antipode $\mathrm{S}^{-1}$ and counit as before. According to Drinfeld [1] a Hopf algebra A is called quasitriangular if there exists an invertible element $R$ such that

$$
\begin{gathered}
R=\sum_{i} a_{i} \otimes b_{i} \in A \otimes A \\
\Delta^{T}(a) R=R \Delta(a) \quad \forall a \in A \\
R_{12} R_{13} R_{23}=R_{23} R_{13} R_{12}
\end{gathered}
$$

where $a_{i}$ and $b_{i}$ are appropriately chosen dual basis of $A, R_{13} R_{23}=(\Delta \otimes I) R, R_{13} R_{12}=(I \otimes \Delta) R$ and $R^{-1}=(S \otimes I) R$. Finally for any Hopf algebra we can define the adjoint operations $a d$ and $a d^{\prime}$ given by

$$
\begin{gathered}
a d_{a}(b)=\sum_{(a)} a^{(1)} b S\left(a^{(2)}\right) \\
a d_{a}^{\prime}(b)=\sum_{(a)} a^{(2)} b S^{-1}\left(a^{(1)}\right) \quad \forall a \in A .
\end{gathered}
$$




\section{The undeformed generalized boson algebras $B_{\alpha, \beta}$ and $\bar{B}_{\sigma, \tau}$.}

We start by recalling that the boson algebra $B$ is generated by $a, a^{\dagger}$, and $N$ subject to the following relations:

$$
\left[a, a^{\dagger}\right]=I, \quad[N, a]=-a, \quad\left[N, a^{\dagger}\right]=a^{\dagger}
$$

and a Fock space representation is provided by

$$
\left|n>=\frac{1}{\sqrt{n !}}\left(a^{\dagger}\right)^{n}\right| 0>, \quad N|n>=n| n>, \quad a|n>=\sqrt{n}| n-1>, \quad a^{\dagger}|n>=\sqrt{(n+1)}| n+1>.
$$

The often used identification $a^{\dagger} a=N$ and $a a^{\dagger}=N+1$ holds in the quotient $B /<C>$ (and on the above Fock space) where $\langle C\rangle$ is the two-sided ideal generated by $C=a^{\dagger} a-N$. As was demonstrated in [40], a Hopf algebra structure on this algebra fails to exist.

\subsection{The algebra $B_{\alpha, \beta}$.}

We shall now consider the family of algebras $B_{\alpha, \beta}$ generated by $a, a^{\dagger}$ and $N$ subject to the following relations:

$$
\begin{aligned}
\left\{a, a^{\dagger}\right\} & =\alpha N+\beta I, \\
{[N, a] } & =-a, \\
{\left[N, a^{\dagger}\right] } & =a^{\dagger}
\end{aligned}
$$

where $\alpha, \beta \in \mathbf{R}$. Here and in the rest of the paper $\{x, y\}=x y+y x$. If we take the quotient of $B_{2,1}$ with respect to an ideal generated by $a^{\dagger} a-N$ we recover $B /\langle C\rangle$ above. This algebra can be enlarged to become a Hopf algebra by adding an invertible element $(-1)^{N}$ which will be treated as a supplementary generator satisfying the following relations:

$$
\left\{(-1)^{N}, a\right\}=0=\left\{(-1)^{N}, a^{\dagger}\right\}, \quad\left[(-1)^{N}, N\right]=0,
$$

Similar considerations were used in [46] and in that paper's context our enlarged algebra $B_{\alpha, \beta}$ can be thought of as a spectrum generating algebra for the ordinary harmonic oscillator, while the element $g$ of [46] will be $g=(-1)^{\widetilde{N}}$ provided that we impose the condition $g^{2}=(-1)^{2 \widetilde{N}}=I$ where $\widetilde{N}=N+\frac{\beta}{\alpha}$. At this point we do not necessarily have to impose this condition (which implies that $\left.(-1)^{2 N}=(-1)^{-\frac{2 \beta}{\alpha}}\right)$. We shall denote by $B_{\alpha, \beta}^{+}$and $U\left(B_{\alpha, \beta}^{+}\right)$, this enlarged algebra and its universal enveloping algebras respectively. Then the coproduct counit and antipode satisfy (đ) are given by:

$$
\begin{gathered}
\Delta(N)=N \otimes I+I \otimes N+\frac{\beta}{\alpha} I \otimes I \\
\Delta(a)=a \otimes I+(-1)^{N+\frac{\beta}{\alpha}} \otimes a \\
\Delta\left(a^{\dagger}\right)=a^{\dagger} \otimes I+(-1)^{-N-\frac{\beta}{\alpha}} \otimes a^{\dagger}
\end{gathered}
$$




$$
\begin{gathered}
\varepsilon(N)=-\frac{\beta}{\alpha}, \quad \varepsilon(a)=\varepsilon\left(a^{\dagger}\right)=0, \quad \varepsilon(I)=1 \\
S(N)=-N-\frac{2 \beta}{\alpha}, \quad S(a)=-(-1)^{-N-\frac{\beta}{\alpha}} a, \quad S\left(a^{\dagger}\right)=a^{\dagger}(-1)^{N+\frac{\beta}{\alpha}}, \\
\Delta\left((-1)^{ \pm \widetilde{N}}\right)=(-1)^{ \pm \widetilde{N}} \otimes(-1)^{ \pm \widetilde{N}}, \quad \varepsilon\left((-1)^{ \pm \widetilde{N}}\right)=I, \quad S\left((-1)^{ \pm \widetilde{N}}\right)=(-1)^{\mp \widetilde{N}} .
\end{gathered}
$$

provided that $\alpha \neq 0$. Moreover an opposite Hopf algebra structure also exists for $B_{\alpha, \beta}$ with coproduct $\Delta^{T}$ and antipode the inverse $S^{-1}$ of $S$ given by :

$$
\begin{aligned}
& S^{-1}(N)=S(N), \quad S^{-1}(a)=(-1)^{-N-\frac{\beta}{\alpha}} a, \\
& S^{-1}\left(a^{\dagger}\right)=-a^{\dagger}(-1)^{N+\frac{\beta}{\alpha}}, \quad S^{-1}\left((-1)^{ \pm \widetilde{N}}\right)=S\left((-1)^{ \pm \widetilde{N}}\right) .
\end{aligned}
$$

A Fock-type representation, with $a|0>=0| n>,^{\prime}=\left(a^{\dagger}\right)^{n}|0>, N| n>^{\prime}=n \mid n>^{\prime}, n \in Z_{+}$and $<0 \mid 0>=1$, exists such that,

$$
a\left|n>^{\prime}=[n]\right| n-1>^{\prime}, \quad \text { where } \quad[n]=\frac{\alpha n}{2}+\frac{2 \beta-\alpha}{4}\left(1+(-1)^{n+1}\right)
$$

If $\alpha>0, \beta>0$ a unitary representation of $B_{\alpha, \beta}$ is provided by:

$$
\begin{gathered}
\left|n>=\frac{1}{([n] !)^{\frac{1}{2}}}\left(a^{\dagger}\right)^{n}\right| 0> \\
a\left|n>=[n]^{\frac{1}{2}}\right| n-1>, \quad a^{\dagger}\left|n>=[n+1]^{\frac{1}{2}}\right| n+1>, \\
\text { where }[n] !=\prod_{l=1}^{n}[l] \quad \text { and } \quad<n \mid n^{\prime}>=\delta_{n n^{\prime}} .
\end{gathered}
$$

With the definition $(-1)^{ \pm N}\left|n>=(-1)^{ \pm n}\right| n>$ this Fock space provides also a representation of $B_{\alpha, \beta}^{+}$.

There exists an element $L$ in the enveloping algebra $U\left(B_{\alpha, \beta}\right)$ of $B_{\alpha, \beta}$ given by

$$
L=\lambda_{1} a^{\dagger} a+\lambda_{2} N+\lambda_{3} I
$$

and such that

$$
\{L, a\}=\left\{L, a^{\dagger}\right\}=0,
$$

provided that the following constraints on $\lambda_{i} \in \mathbf{R}(i=1,2,3)$ are satisfied

$$
2 \lambda_{3}+\beta \lambda_{1}+\lambda_{2}=0, \quad 2 \lambda_{2}+\alpha \lambda_{1}=0,
$$

(these will give for example $\lambda_{2} / \lambda_{1}=-\alpha / 2$, and $\lambda_{3} / \lambda_{1}=(\alpha-2 \beta) / 4=-\alpha / 2\left(\lambda_{3} / \lambda_{2}\right)$, with $\alpha \neq 0$ ). This choice of $L$ subject to (25) is obviously not unique as it can be easily checked that any odd power of $L$ will satisfy (24). However (23) is the unique element of linear combination of lowest order monomials of generators of $B_{\alpha, \beta}$ that will satisfy (24). This can be inferred by 
writing $L^{\prime}=C_{l m n} K^{l}\left(a^{\dagger}\right)^{m} a^{n}, l, m, n \in \mathbf{Z}_{+}, C_{l m n} \in \mathbf{R}$ and demanding that (24) are satisfied together with $\left[L^{\prime}, N\right]=0$. For a given $B_{\alpha, \beta}$, i.e. for given values of $\alpha$ and $\beta$ relations (25) give us the conditions on $\lambda_{i}$ under which $L$ becomes zero. On what follows we shall assume unless otherwise stated that $L$ is non-zero (e.g. when $\alpha=0$ and $\beta=0$ then $L \neq 0$ if and only if $\lambda_{1} \neq 0$ or when $\alpha=0$ and $\beta \neq 0$ then $L \neq 0$ if and only if $\beta \lambda_{1}=-2 \lambda_{3} \neq 0$ ). Then using (25), $L \in U\left(B_{\alpha, \beta}\right)$ can be put in the form

$$
L=\lambda_{1}\left(a^{\dagger} a-\frac{\alpha}{2} N+\left(\frac{\alpha}{4}-\frac{\beta}{2}\right) I\right), \quad \lambda_{1} \neq 0 .
$$

If we consider $B_{\alpha, \beta}^{+}$then the additional term $\lambda_{4}(-1)^{\widetilde{N}}\left(\lambda_{4} \in \mathbf{R}, \lambda_{4} \neq 0\right)$ can be considered and an element $L^{+} \in U\left(B_{\alpha, \beta}^{+}\right)$can be taken as

$$
L^{+}=L+\lambda_{4}(-1)^{\widetilde{N}}
$$

satisfying (24), while for given values of $\alpha$ and $\beta, L^{+}$is also not unique and odd powers of it will give (24). However it should be noted that in the case of $B_{\alpha, \beta}^{+}$the element $\lambda_{4}(-1)^{\widetilde{N}}$ is the unique non-zero lowest order monomial satisfying (24). This again can be inferred by writing $L^{\prime+}=C_{p l m n}(-1)^{p N} K^{l}\left(a^{\dagger}\right)^{m} a^{n}, p, l, m, n \in \mathbf{Z}_{+}, C_{p l m n} \in \mathbf{R}$ and demanding that (24) are satisfied together with $\left[L^{\prime+}, N\right]=0$. Relation (27) is then the next most general one to be considered. Utilizing (18) and (25), it can be found that $\Delta\left(L^{+}\right), S\left(L^{+}\right)$and $\varepsilon\left(L^{+}\right)$are given by

$$
\begin{aligned}
\Delta\left(L^{+}\right)= & L \otimes I+I \otimes L-\lambda_{1} \frac{\alpha}{4} I \otimes I+\lambda_{4}(-1)^{\widetilde{N}} \otimes(-1)^{\widetilde{N}} \\
& -\lambda_{1}\left((-1)^{\widetilde{N}} a^{\dagger} \otimes a-(-1)^{-\widetilde{N}} a \otimes a^{\dagger}\right) \\
S\left(L^{+}\right)= & L^{+}, \quad \varepsilon\left(L^{+}\right)=\lambda_{1} \frac{\alpha}{4}+\lambda_{4} .
\end{aligned}
$$

and that (7) are satisfied. Specialization to include only $L$ in the above relations, can be obtained by setting $L^{+} \rightarrow L$ and $\lambda_{4}=0$. Relations (24) are also preserved by the Hopf algebra structure (18), subject to (25), while $L^{+}$is represented on the Fock space (22) as

$$
L^{+}\left|n>=\left(\lambda_{1}\left(\frac{\alpha}{4}-\frac{\beta}{2}\right)+\lambda_{4}(-1)^{\frac{\beta}{\alpha}}\right)(-1)^{n}\right| n>
$$

Note that $L^{+}$(and $L$ ) introduces a $Z_{2}$ grading on the Fock space.

As mentioned the constraints (25) can be widely exploited leading to various choices of values for $\lambda_{i}$ in terms of $\alpha, \beta$. In the case $\alpha=2$ and $\beta=1, \lambda_{1}=-\lambda_{2}, \lambda_{3}=0$ and $L^{+}=$ $\lambda_{1}\left(a^{\dagger} a-N\right)+\lambda_{4}(-1)^{\widetilde{N}}$. Then (22) show that on this Fock space $a^{\dagger} a=N, a a^{\dagger}=N+I$ and $B_{2,1}^{+}$reduces to the quotient $B /<C>$ (see beginning of the section) extended with the element $(-1)^{a^{\dagger} a+1 / 2}$. Also we can investigate the case where we impose on $B_{\alpha, \beta}$ (or $B_{\alpha, \beta}^{+}$) the additional relation

$$
L^{2}=\eta I, \quad\left(\text { or }\left(L^{+}\right)^{2}=\eta I\right)
$$

with $\eta \in \mathbf{R}, \eta \neq 0$. From the form of $L$ (26) it can be easily observed that $L^{2}$ commutes with all the generators of $B_{\alpha, \beta}$ (and $B_{\alpha, \beta}^{+}$) and thus on any faithful representation it reduces to a multiple of the identity. Also it can be shown that (31) does not respect the Hopf algebra structure. On a representation independent way thought using (26) we obtain the characteristic identity for $C=a^{\dagger} a-\frac{\alpha}{2} N$ 


$$
C\left(C+\left(\frac{\alpha}{2}-\beta\right) I\right)+\left(\frac{\alpha}{4}-\frac{\beta}{2}\right)^{2} I=\frac{\eta}{\lambda_{1}^{2}} I
$$

which when solved will give a $L$ as a multiple of the identity. Obviously (31) with the choice of $L$ given by (26), is not compatible with relations (24). The same incompatibility is also true for the case of $B_{\alpha, \beta}^{+}$and $L^{+}$given in (27). However if we consider the element $\lambda_{4}(-1)^{\widetilde{N}}=L^{+}$alone (thus letting $\lambda_{1}=0$ ) then (31) can hold (it is just imposing the requirement $g^{2}=I$ ) and the Hopf algebra is preserved.

Finally it is important in what will follow in section 5 to observe that if we substitute in (16) the generators $N$ obtained from (26) (or (27) ) as $N=\frac{2}{\alpha}\left(-\frac{1}{\lambda_{1}} L+a^{\dagger} a+\frac{\alpha}{4}-\frac{\beta}{2}\right)$, then (16) becomes

$$
\left[a, a^{\dagger}\right]=-\frac{2}{\lambda_{1}} L+\frac{\alpha}{2} I
$$

This is true if and only if the values of $\alpha$ and $\beta$ are such that $L$ and $L^{+}$contain the monomial $N$, that is when the following values of the pair $(\alpha, \beta)$ are not considered: $\alpha=0, \beta=0$ and $\alpha=0, \beta \neq 0$. Relation (33) shows the potentiality of $B_{\alpha, \beta}$ (and $B_{\alpha, \beta}^{+}$) to accommodate and interchange both commutation and anticommutation relations. It is interesting to investigate if this relation together with (24) serve as an alternative definition of $B_{\alpha, \beta}\left(\right.$ or $\left.B_{\alpha, \beta}^{+}\right)$. This will become clearer in section 5 where (33) and (24) will be compared with (59).

We can obtain from $B_{\alpha, \beta}$ a realization of $B(0 / 1) \simeq \operatorname{osp}(1 / 2)$ by introducing a $\mathbf{Z}_{2}$ grading such that $a$ and $a^{\dagger}$ are odd and $N$ is even and defining

$$
e=\mu a^{\dagger}, \quad f=\lambda a, \quad h=2 N+\frac{2 \beta}{\alpha} I
$$

provided $\alpha \neq 0$ and $\mu \lambda=\frac{2}{\alpha}$, so that

$$
\{e, f\}=h, \quad[h, e]=2 e, \quad[h, f]=-2 f .
$$

Then the Hopf algebra structure of $B_{\alpha, \beta}$ induces a non-trivial one for $\operatorname{ssp}(1 / 2)$ extended by the element $g=(-1)^{\widetilde{N}}$, exactly as in the case of [46] (but using $B_{\alpha, \beta}$ instead of the ordinary oscillator algebra) and the $R$-matrix is given by (52) below. The Casimir invariant $I_{2}=-\frac{1}{4} e^{2} f^{2}-$ $\frac{1}{4} e f+\frac{1}{16} h^{2}-\frac{1}{8} h$ on the Fock space (22) takes the eigenvalue

$$
i_{2}=\frac{\beta^{2}}{4 \alpha^{2}}-\frac{\beta}{4 \alpha}
$$

which shows that the representation is irreducible. Similarly we can obtain a realization of $A_{1}$ (as a subalgebra of $B(0 / 1)$ for example) by defining

$$
e^{\prime}=\mu^{\prime} e^{2}, \quad f^{\prime}=\lambda^{\prime} f^{2}, \quad h^{\prime}=\frac{1}{2} h
$$

provided $\mu^{\prime} \lambda^{\prime}=-\frac{1}{4}$, so that

$$
\left[e^{\prime}, f^{\prime}\right]=h^{\prime}, \quad\left[h^{\prime}, e^{\prime}\right]=2 e^{\prime}, \quad\left[h^{\prime}, f^{\prime}\right]=-2 f^{\prime} .
$$


We can also obtain a realization of $\operatorname{sl}(2, R) \simeq s u(1,1)$ if we set $J_{0}=\frac{1}{2} h^{\prime}, J_{+}=\frac{i}{\sqrt{2}} e^{\prime}$ and $J_{-}=\frac{i}{\sqrt{2}} f^{\prime}$. Then the eigenvalues of the $s l(2, R)$ Casimir invariant $C_{2}=2 J_{-} J_{+}-J_{0}^{2}-J_{0}$ on the Fock space (22) are given by

$$
c_{n}=\frac{1}{2}-\frac{\beta}{2 \alpha}-\frac{\beta^{2}}{4 \alpha^{2}}+\frac{2 \beta-\alpha}{8 \alpha}\left(3+(-1)^{n}\right) .
$$

which shows that the representation is completely reducible with the two invariant subspaces corresponding to $n$ being even and $n$ being odd. The Casimir eigenvalues $c_{\text {even }}$ and $c_{\text {odd }}$ are

$$
c_{\text {even }}=-\frac{1}{4} \frac{\beta^{2}}{\alpha^{2}}+\frac{\beta}{2 \alpha}, \quad c_{o d d}=\frac{1}{4}-\frac{\beta^{2}}{4 \alpha^{2}} .
$$

\subsection{The algebra $\bar{B}_{\sigma, \tau}$.}

We shall consider now the family of algebras $\bar{B}_{\sigma, \tau}$ generated by $a, a^{\dagger}$ and $N$ subject to the following relations:

$$
\begin{aligned}
{\left[a, a^{\dagger}\right] } & =\sigma N+\tau I \\
{[N, a] } & =-a, \\
{\left[N, a^{\dagger}\right] } & =a^{\dagger}
\end{aligned}
$$

where $\sigma, \tau \in \mathbf{R}$ and for $\sigma=0$ we obtain (14). This algebra is a Hopf algebra whose coproduct counit and antipode satisfying (7) are given by:

$$
\begin{gathered}
\Delta(N)=N \otimes I+I \otimes N+\frac{\tau}{\sigma} I \otimes I \\
\Delta(a)=a \otimes I+I \otimes a \\
\Delta\left(a^{\dagger}\right)=a^{\dagger} \otimes I+I \otimes a^{\dagger} \\
\varepsilon(N)=-\frac{\tau}{\sigma}, \varepsilon(a)=\varepsilon\left(a^{\dagger}\right)=0, \varepsilon(I)=1 \\
S(N)=-N-\frac{2 \tau}{\sigma}, \quad S(a)=-a, \quad S\left(a^{\dagger}\right)=-a^{\dagger}
\end{gathered}
$$

provided $\sigma \neq 0$ (thus not allowing a Hopf algebra structure for (14). An opposite Hopf algebra structure can be easily read off from (40) which also show that an $R$-matrix will turn out to be trivial. A Fock-type representation, with $a|0>=0| n>,^{\prime}=\left(a^{\dagger}\right)^{n}|0>, N| n>^{\prime}=n \mid n>^{\prime}, n \in Z_{+}$ and $<0|0\rangle=1$, exists such that

$$
a\left|n>^{\prime}=\overline{[n]}\right| n-1>^{\prime}, \quad \text { where } \overline{[n]}=\frac{\sigma n(n-1)}{2}+n \tau .
$$

If $\sigma \geq 0, \tau \geq 0$ a unitary representation of $\bar{B}_{\sigma, \tau}$ is provided by:

$$
\left|n>=\frac{1}{(\overline{[n] !})^{\frac{1}{2}}}\left(a^{\dagger}\right)^{n}\right| 0>
$$




$$
\begin{gathered}
a\left|n>=\overline{[n]^{\frac{1}{2}}}\right| n-1>, \quad a^{\dagger}\left|n>=\overline{[n+1]^{\frac{1}{2}}}\right| n+1>, \\
\quad \text { where } \overline{[n] !}=\prod_{l=1}^{n} \overline{[l]} \quad \text { and } \quad<n \mid n^{\prime}>=\delta_{n n^{\prime}} .
\end{gathered}
$$

Finally an $A_{1}$ realization can be obtain by setting

$$
e=\xi a^{\dagger}, \quad f=\zeta a, \quad h=2 N+\frac{2 \tau}{\sigma} I
$$

where $\xi \zeta=-2 / \sigma$ and the defining relations of $A_{1}$ are as shown below (37).

\section{Deformed Boson algebras $B_{\alpha, \beta}^{q}$ and $\bar{B}_{\sigma, \tau}^{q}$.}

\subsection{The algebra $B_{\alpha, \beta}^{q}$.}

We turn now to a $q$-deformation ( $q$ generic) of the algebras $B_{\alpha, \beta}$ and $\bar{B}_{\sigma, \tau}$. Define $B_{\alpha, \beta}^{q}$ as the Lie algebra generated by $a_{q}, a_{q}^{\dagger}$ and $N$ subject to the following relations:

$$
\begin{aligned}
\left\{a_{q}, a_{q}^{\dagger}\right\} & =[\alpha N+\beta]_{q} \\
{\left[N, a_{q}\right] } & =-a_{q} \\
{\left[N, a_{q}^{\dagger}\right] } & =a_{q}^{\dagger}
\end{aligned}
$$

where $\alpha, \beta \in \mathbf{R}$ and $[x]_{q}=\left(q^{x}-q^{-x}\right) /\left(q-q^{-1}\right)$. This algebra is a Hopf algebra whose coproduct, counit and antipode satisfy (77) and are given by:

$$
\begin{gathered}
\Delta(N)=N \otimes I+I \otimes N+\frac{\beta}{\alpha} I \otimes I \\
\Delta(a)=a_{q} \otimes q^{\frac{\alpha N+\beta}{2}}+(-1)^{N+\frac{\beta}{\alpha}} q^{-\frac{\alpha N+\beta}{2}} \otimes a_{q} \\
\Delta\left(a_{q}^{\dagger}\right)=a_{q}^{\dagger} \otimes q^{\frac{\alpha N+\beta}{2}}+(-1)^{-N-\frac{\beta}{\alpha}} q^{-\frac{\alpha N+\beta}{2}} \otimes a_{q}^{\dagger} \\
\varepsilon(N)=-\frac{\beta}{\alpha}, \quad \varepsilon\left(a_{q}\right)=\varepsilon\left(a_{q}^{\dagger}\right)=0, \quad \varepsilon(I)=1 \\
S(N)=-N-\frac{2 \beta}{\alpha}, \quad S\left(a_{q}\right)=-(-1)^{-N-\frac{\beta}{\alpha}} q^{-\frac{\alpha}{2}} a, \quad S\left(a_{q}^{\dagger}\right)=a_{q}^{\dagger}(-1)^{N+\frac{\beta}{\alpha}} q^{\frac{\alpha}{2}}
\end{gathered}
$$

provided that $\alpha \neq 0$. An opposite Hopf algebra structure also exists with coproduct $\Delta^{T}$ and antipode the inverse $S^{-1}$ of $S$ given by :

$$
S^{-1}(N)=S(N), \quad S^{-1}\left(a_{q}\right)=(-1)^{-N-\frac{\beta}{\alpha}} q^{-\frac{\alpha}{2}} a_{q}, \quad S^{-1}\left(a_{q}^{\dagger}\right)=-a_{q}^{\dagger}(-1)^{N+\frac{\beta}{\alpha}} q^{\frac{\alpha}{2}} .
$$

Similarly to the undeformed case, in order to obtain (45), we have to enlarge $B_{\alpha, \beta}^{q}$ by adding an invertible element $(-1)^{\widetilde{N}}$ which will be treated as an supplementary generator satisfying 
relations (17) (with $a_{q}$ and $a_{q}^{\dagger}$ in the place of $a$ and $a^{\dagger}$ respectively) and (19). We shall denote this extended algebra (its universal enveloping algebra) as $B_{\alpha, \beta}^{q+}\left(U\left(B_{\alpha, \beta}^{q+}\right)\right)$.

A Fock-type representation, with $a_{q}\left|0>_{q}=0,\right| n>_{q}^{\prime}=\left(a_{q}^{\dagger}\right)^{n}\left|0>_{q}, N\right| n>_{q}=n \mid n>_{q}, n \in Z_{+}$ and ${ }_{q}<0 \mid 0>_{q}=1$, exists such that

$$
\begin{gathered}
a_{q}\left|n>_{q}^{\prime}=(n)_{q}\right| n-1>_{q}^{\prime}, \quad(-1)^{ \pm N}\left|n>_{q}^{\prime}=(-1)^{ \pm n}\right| n>_{q}^{\prime}, \\
\text { where }(n)_{q}=\left(q^{\frac{\alpha}{2}}+q^{-\frac{\alpha}{2}}\right)^{-1}\left((-1)^{n+1}\left[\beta-\frac{\alpha}{2}\right]_{q}+\left[n \alpha+\beta-\frac{\alpha}{2}\right]_{q}\right)
\end{gathered}
$$

Normalizing $\mid n>_{q}^{\prime}$ the representation of $B_{\alpha, \beta}^{q}\left(\right.$ and $\left.B_{\alpha, \beta}^{q+}\right)$ is provided by:

$$
\begin{gathered}
\left|n>_{q}=\frac{1}{\left((n)_{q} !\right)^{\frac{1}{2}}}\left(a_{q}^{\dagger}\right)^{n}\right| 0>_{q} \\
a_{q}\left|n>_{q}=(n)_{q}^{\frac{1}{2}}\right| n-1>_{q}, \quad a_{q}^{\dagger}\left|n>_{q}=(n+1)_{q}^{\frac{1}{2}}\right| n+1>_{q}, \\
\text { where } \quad(n)_{q} !=\Pi_{m=1}^{n}(m)_{q} \quad \text { and } \quad{ }_{q}<n \mid n^{\prime}>_{q}=\delta_{n n^{\prime}} .
\end{gathered}
$$

In the limit $q \rightarrow 1$ we get the Fock space of the undeformed algebra $B_{\alpha, \beta}\left(\right.$ or $\left.B_{\alpha, \beta}^{+}\right)$.

A realization of $\operatorname{ssp}_{q^{\prime}}(1 / 2)$, with $q^{\prime}=q^{\alpha}$, can be obtained by defining

$$
e=\mu a_{q}^{\dagger}, \quad f=\lambda a_{q}, \quad h=N+\frac{\beta}{\alpha} I
$$

so that with $\mu \lambda=[\alpha]_{q}^{-1}$ the following $\operatorname{osp}_{q^{\prime}}(1 / 2)$ defining relations are satisfied:

$$
\{e, f\}=[h]_{q^{\prime}}, \quad[h, e]=e, \quad[h, f]=-f .
$$

Finally an $R$-matrix exists and is given by

$$
R=R_{0} q^{\alpha \widetilde{N} \otimes \widetilde{N}} \sum_{l=0}^{\infty}\left(q-q^{-1}\right)^{l} q^{-\frac{\alpha}{4} l(l+1)} \frac{(-1)^{\frac{1}{4} l(l-1)}}{[l]_{x} !} q^{\frac{\alpha}{2} l \widetilde{N}}(-1)^{l \widetilde{N}}\left(a_{q}^{\dagger}\right)^{l} \otimes q^{-\frac{\alpha}{2} l \widetilde{N}} a_{q}^{l}
$$

where $\tilde{N}=N+\beta / \alpha, x=\left(-q^{-\alpha}\right)^{1 / 2}$ and

$$
R_{0}=\frac{1}{2}\left(I \otimes I+I \otimes(-1)^{\widetilde{N}}+(-1)^{\widetilde{N}} \otimes I-(-1)^{\widetilde{N}} \otimes(-1)^{\widetilde{N}}\right)
$$

provided that we demand that $(-1)^{2 \widetilde{N}}=I$. (9) has been calculated using quantum double technics similar to [44. It is important to mention that (9) is exactly the same as the one appeared in relation (49) in 46] with $q \rightarrow q^{\alpha}$, provided we do the following identifications with the generators of the bosonization of $\operatorname{osp}_{q^{\alpha}}(1 / 2): J_{z}=\frac{1}{2} \widetilde{N}, V_{+}=k a_{q}^{\dagger}, V_{-}=t a_{q}, g=(-1)^{\widetilde{N}}$, and $k t=-[4]_{q^{\alpha}}^{-1}[\alpha]_{q}^{-1}$. Then we can argue that $B_{\alpha, \beta}^{q+}$ is the spectrum generating quantum group for the ordinary $q$-deformed harmonic oscillator defined by the relations $a_{q} a_{q}^{\dagger}-q^{ \pm} a_{q}^{\dagger} a_{q}=q^{\mp N}$ and the last two of (44), which as will be shown in [45] is a subalgebra of $B_{\alpha, \beta}^{q+}$. At the limit $q \rightarrow 1, R \rightarrow R_{0}$ which is the $R$ - matrix of the undeformed $B_{\alpha, \beta}^{+}$. 


\subsection{The algebra $\bar{B}_{\sigma, \tau}^{q}$.}

Similarly define $\bar{B}_{\sigma, \tau}^{q}$ as the Lie algebra generated by $a_{q}, a_{q}^{\dagger}$ and $N$ subject to the following relations:

$$
\begin{aligned}
& {\left[a_{q}, a_{q}^{\dagger}\right]=[\sigma N+\tau I]_{q}} \\
& {\left[N, a_{q}\right]=-a_{q},} \\
& {\left[N, a_{q}^{\dagger}\right]=a_{q}^{\dagger}}
\end{aligned}
$$

where $\sigma, \tau \in \mathbf{R}$.

This algebra is a Hopf algebra whose coproduct, counit and antipode satisfy (而) and are given by:

$$
\begin{gathered}
\Delta(N)=N \otimes I+I \otimes N+\frac{\tau}{\sigma} I \otimes I, \quad \lambda \neq 0 \\
\Delta\left(a_{q}\right)=a_{q} \otimes q^{\frac{\sigma N+\tau}{2}}+q^{-\frac{\sigma N+\tau}{2}} \otimes a_{q} \\
\Delta\left(a_{q}^{\dagger}\right)=a_{q}^{\dagger} \otimes q^{\frac{\sigma N+\tau I}{2}}+q^{-\frac{\sigma N+\tau}{2}} \otimes a_{q}^{\dagger} \\
\varepsilon(N)=-\frac{\tau}{\sigma}, \quad \varepsilon\left(a_{q}\right)=\varepsilon\left(a_{q}^{\dagger}\right)=0, \quad \varepsilon(I)=1 \\
S(N)=-N-\frac{2 \tau}{\sigma}, \quad S\left(a_{q}\right)=-q^{-\frac{\sigma}{2}} a_{q}, \quad S\left(a_{q}^{\dagger}\right)=-q^{\frac{\sigma}{2}} a_{q}^{\dagger} .
\end{gathered}
$$

An opposite Hopf algebra structure exists whose antipode $S^{-1}$ is given by

$$
S^{-1}(N)=S(N), \quad S^{-1}\left(a_{q}\right)=-q^{\frac{\sigma}{2}} a_{q}, \quad S^{-1}\left(a_{q}^{\dagger}\right)=-q^{-\frac{\sigma}{2}} a_{q}^{\dagger}
$$

A Fock-type representation, with $a_{q}\left|0>_{q}=0,\right| n>_{q}^{\prime}=\left(a_{q}^{\dagger}\right)^{n}\left|0>_{q} N\right| n>_{q}^{\prime}=n \mid n>_{q}^{\prime}, n \in Z_{+}$, and ${ }_{q}<0 \mid 0>_{q}=1$, exists such that normalizing $\mid n>_{q}^{\prime}$ we get

$$
\begin{gathered}
\left|n>_{q}=\frac{1}{\left.\overline{(n)_{q}} !\right)^{\frac{1}{2}}}\left(a_{q}^{\dagger}\right)^{n}\right| 0>_{q} \\
a_{q}\left|n>_{q}=\overline{(n)_{q}^{\frac{1}{2}}}\right| n-1>_{q}, \quad a_{q}^{\dagger}\left|n>_{q}=\overline{(n+1)_{q}^{\frac{1}{2}}}\right| n+1>_{q} \\
\overline{(n)}_{q}=\left[\frac{\sigma}{2}\right]_{q}^{-1}\left(\left[\frac{\sigma n}{2}\right]_{q}\left[\frac{\sigma(n-1)}{2}+\tau\right]_{q}\right) \\
{\overline{(n)_{q}}}_{q}=\Pi_{m=1}^{n}{\overline{(m)_{q}}}_{q} \text { and } \quad{ }_{q}<n \mid n^{\prime}>_{q}=\delta_{n n^{\prime}}
\end{gathered}
$$

In the limit $q \rightarrow 1$ we get the Fock space of the undeformed algebra $\bar{B}_{\sigma, \tau}$. A $s l_{q^{\sigma / 2}}(2)$ realization is provided by the following identifications 


$$
e=\xi a_{q}^{\dagger}, \quad f=\zeta a_{q}, \quad h=2 N+\frac{2 \tau}{\sigma} I
$$

where $\xi \zeta=-\left[\frac{\sigma}{2}\right]_{q}$, so that the defining $s l_{q^{\sigma / 2}}(2)$ relations below are satisfied:

$$
[e, f]=[h]_{q^{\sigma / 2}}, \quad[h, e]=2 e, \quad[h, f]=-2 f .
$$

An $R$-matrix also exists, and is given by

$$
R=q^{\sigma \widetilde{N} \otimes \widetilde{N}} \sum_{l=0}^{\infty} q^{\frac{\sigma}{4} l(l+1)} \frac{(-1)^{l}}{[l]_{x} !}\left(q-q^{-1}\right)^{l} q^{\frac{\sigma}{4} l \widetilde{N}}\left(a_{q}^{\dagger}\right)^{l} \otimes q^{-\frac{\sigma}{4} l \widetilde{N}} a_{q}^{l}
$$

where $\widetilde{N}=N+\frac{\tau}{\sigma}$ and $x=\left(q^{\sigma / 2}\right)$. As in the case of (9), (10) has also been obtained using quantum double technic.

\section{The generalized ' $\nu$-deformed' Heisenberg algebra $\mathbf{H}_{\delta, \nu}$.}

We shall generalize now the so-called 'deformed' Heisenberg algebra of Vassiliev [12]. This is defined as the algebra $H_{\delta, \nu}$ generated by $b, b^{\dagger}$ and $K$ subject to the following relations:

$$
\begin{aligned}
{\left[b, b^{\dagger}\right] } & =\delta I+\nu K \\
\{K, b\} & =\left\{K, b^{\dagger}\right\}=0
\end{aligned}
$$

where $\delta, \nu \in \mathbf{R}$. If we impose the additional requirements that $K^{2}=I$ then with $\delta=1$ we obtain that of [12], used for example in [13], [29], [30].

A Fock-type representation ( a generalization of that appearing in [29], 30] ), with $b \mid 0>=0$, $\left|n>^{\prime}=\left(b^{\dagger}\right)^{m}\right| 0>, m \in \mathbf{Z}_{+}$and $<0 \mid 0>=1$, exists such that

$$
b\left|n>^{\prime}=[m]\right| m-1>^{\prime}, \quad \text { where } \quad[m]=\delta m+\frac{\nu-\delta+1}{2}\left(1+(-1)^{m+1}\right)
$$

If $\nu>0$ and $\delta>0$ a unitary representation of $B_{\alpha, \beta}$ is provided by:

$$
\begin{gathered}
\left|m>=\frac{1}{([m] !)^{\frac{1}{2}}}\left(b^{\dagger}\right)^{m}\right| 0>, \\
b\left|m>=[m]^{\frac{1}{2}}\right| m-1>, \quad b^{\dagger}\left|m>=[m+1]^{\frac{1}{2}}\right| m+1>, \\
K\left|m>=\frac{\nu-\delta+1}{\nu}(-1)^{m}\right| m>, \\
{[m] !=\prod_{l=1}^{m}[l], \quad<m \mid m^{\prime}>=\delta_{m m^{\prime}} .}
\end{gathered}
$$

The striking similarity of the Fock spaces (22) and (61) is not accidental. As we shall just show under certain conditions we can obtain $B_{\alpha, \beta}$ from $H_{\delta, \nu}$ and vice versa, not only on the above Fock spaces but as abstract algebras. $H_{\delta, \nu}$ can be extended so that the resulting algebra will possess a Hopf algebra structure. There exists in the enveloping algebra $U\left(H_{\delta, \nu}\right)$ of $H_{\delta, \nu}$, an element $M$ given by 


$$
M=\mu_{1} b^{\dagger} b+\mu_{2} K+\rho I
$$

and satisfying

$$
[M, b]=-b, \quad\left[M, b^{\dagger}\right]=b^{\dagger},
$$

where $\mu_{i}, \rho \in \mathbf{R}(i=1,2)$ provided that the following constraint is satisfied

$$
\mu_{1} \delta I+\left(2 \mu_{2}-\nu \mu_{1}\right) K=I .
$$

This suggests that, since $K$ should not be a multiple of the identity, (as this contradicts the second of (59)) necessarily $\delta \neq 0$ which leads to $\mu_{1}=1 / \delta$ and $2 \mu_{2}-\nu \mu_{1}=0$. Consequently with the above constraints (62) now becomes

$$
\begin{gathered}
M=\frac{1}{\delta} b^{\dagger} b+\frac{\nu}{2 \delta} K+\rho I, \\
\text { and } M\left|m>=\left(m+\frac{\nu-\delta+1}{2 \delta}+\rho\right)\right| m>
\end{gathered}
$$

which by choosing $\rho=-\frac{\nu-\delta+1}{2 \delta}, M|m>=m| m>$. The choice of $M$ given by (65) is obviously not the most general possible but it is the unique non-zero combination of lowest order monomials of generators of $H_{\delta, \nu}$ that satisfy (63) while such an element does not exist if $\delta=0$ (these considerations can be inferred by writing $M^{\prime}=C_{l m n} K^{l}\left(b^{\dagger}\right)^{m} b^{n}, l, m, n \in \mathbf{Z}_{+}, C_{l m n} \in \mathbf{R}$, and demanding that (63) are satisfied together with $\left.\left[M^{\prime}, K\right]=0\right)$. Now we are in a position to demonstrate the similarities between $B_{\alpha, \beta}$ and $H_{\delta, \nu}$. Solving (65) with respect to $K$ and substituting in the first of $(59)$ we obtain

$$
\left\{b, b^{\dagger}\right\}=2 \delta M+\delta(1-2 \rho) I
$$

so that together with (63) $H_{\delta, \nu}$ takes the form of the defining relations of $B_{\alpha, \beta}$, (16) by setting

$$
\alpha=2 \delta, \quad \beta=\delta-2 \rho \delta
$$

and where $M$ is replaced by $N$. Note that for the case where we choose $\rho=-\frac{\nu-\delta+1}{2 \delta}, H_{\delta, \nu}$ takes the form of $B_{2 \delta, \nu+1}$. This process can also be carried out the opposite direction, as (24) and (33) suggests, by setting in (33)

$$
\delta=\alpha / 2, \quad \nu=-2 / \lambda_{1}
$$

and where $L$ is replaced by $K$. It is easy to observe that, as $\lambda_{1} \neq 0,(69)$ shows that $B_{\alpha, \beta}$ can not be mapped to a $H_{\delta, 0}$-form. Also $B_{0, \beta}$ can not be mapped to a $H_{\delta, \nu}$-form at all, since the appropriate $L$ fails to exist (no monomial $N$ is present in $L$ even if we perform a thorough search for a more general $L$ in $\left.U\left(B_{\alpha, \beta}\right)\right)$. Also (68) shows that a $B_{\alpha, \beta}$-form of $H_{0, \nu}$ fails to exist since $M$ can not be defined and for $H_{\delta, 0}$ the appropriate $M$ does not exist (no monomial $K$ is present in $M$ ) thus also not allowing a $B_{\alpha, \beta}$-form. Consequently provided that we keep away from the values $\alpha=\delta=\nu=0$ we can always obtain a $H_{\delta, \nu}$-form of $B_{\alpha, \beta}$ and vice-versa. Relations (68) and (69) also imply that $\rho$ and $\beta$ can have arbitrary values. However observation of the Fock spaces (22) and (61) and comparison of the action of $K, M, L$ and $N$ on them, shows that with the identifications (68), (69), $\rho=-\frac{\nu-\delta+1}{2 \delta}, \beta=\nu+1$ not only these spaces are equivalent but also $H_{\delta, \nu}$ and $B_{\alpha, \beta}$ are isomorphic with $K \equiv L, N \equiv M, b \equiv a, b^{\dagger} \equiv a^{\dagger}$.

It can be checked that the following maps $\varphi: B_{\alpha, \beta} \rightarrow H_{\delta, \nu}$ and $\varphi^{\prime}: H_{\delta, \nu} \rightarrow B_{\alpha, \beta}$ defined by: 


$$
\begin{gathered}
\varphi(a)=b, \quad \varphi\left(a^{\dagger}\right)=b^{\dagger}, \quad \varphi(N)=\frac{2}{\alpha} b^{\dagger} b+\frac{\nu}{\alpha} K+\frac{\delta-\beta}{\alpha}, \quad \alpha \neq 0, \\
\varphi^{\prime}(b)=a, \quad \varphi^{\prime}\left(b^{\dagger}\right)=a^{\dagger}, \quad \varphi^{\prime}(K)=-\frac{2}{\nu} a^{\dagger} a+\frac{\alpha}{\nu} N+\frac{\beta-\delta}{\nu}, \quad \nu \neq 0
\end{gathered}
$$

are homomorphisms if and only if $\alpha=2 \delta$. Moreover $\varphi^{\prime}=\varphi^{-1}$ and $\varphi$ becomes an isomorphism $H_{\delta, \nu} \simeq B_{\alpha, \beta}$ provided that both $\alpha \neq 0$ and $\nu \neq 0 . \varphi$ and $\varphi^{\prime}$ can be thought as defining families of maps where each member is parametrized by $\alpha, \beta, \nu$ and $\delta, \beta, \nu$ respectively and we can formally write $\varphi \equiv \varphi_{\alpha, \beta, \nu}$ and $\varphi^{\prime} \equiv \varphi_{\delta, \nu, \beta}^{\prime}$. So for example $B_{2,1}$ is mapped via $\varphi_{2,1, \nu}$ to $H_{1, \nu}$ ( $\nu$ a fixed chosen number) and $H_{1, \nu}$ is mapped via $\varphi_{2,1, \nu}^{-1}=\varphi_{1, \nu, 1}$ back to $B_{2,1}$. Finally it can be checked that

$$
\varphi(L)=-\frac{\lambda_{1} \nu}{2} K, \quad \varphi^{\prime}(M)=N+\frac{\beta-\delta}{2 \delta}+\rho .
$$

With the existence of $M$ of the form (65) we can enlarge $H_{\delta, \nu}$ by adding the invertible element $(-1)^{M}$, as we did in the case of $B_{\alpha, \beta}$. We shall denote this enlarged algebra by $H_{\delta, \nu}^{+}$and the relations that $(-1)^{M}$ has to satisfy are given by:

$$
\left\{(-1)^{ \pm M}, a\right\}=\left\{(-1)^{ \pm M}, a^{\dagger}\right\}=0, \quad\left[(-1)^{ \pm M}, K\right]=0,
$$

and on (61) will be represented as $(-1)^{M}\left|m>=(-1)^{m+\frac{\nu-\delta+1}{2 \delta}+\rho}\right| m>. H_{\delta, \nu}^{+}$can obviously be treated in the spirit of 46] as was done with $B_{\alpha, \beta}^{+}$with the element $g$ of 46 being $g=(-1)^{\widetilde{M}}$, where $\widetilde{M}=M-\rho+\frac{1}{2}$. Thus $H_{\delta, \nu}^{+}$can be considered as a spectrum generating algebra of the ordinary oscillator algebra. Moreover the isomorphism can also be extended such that $H_{\delta, \nu}^{+}$ $\simeq B_{\alpha, \beta}^{+}$by defining $\varphi\left((-1)^{\widetilde{N}}\right)=(-1)^{\widetilde{M}}, \varphi^{\prime}\left((-1)^{\widetilde{M}}\right)=(-1)^{\widetilde{N}}$.

Then a Hopf algebra structure for $H_{\delta, \nu}^{+}$is given by:

$$
\begin{aligned}
& \Delta(K)=K \otimes I+I \otimes K+\frac{\delta}{\nu} I \otimes I \\
& -\frac{2}{\nu}(-1)^{-M+\rho-\frac{1}{2}} b \otimes b^{\dagger}+\frac{2}{\nu}(-1)^{M-\rho+\frac{1}{2}} b^{\dagger} \otimes b \\
& \Delta(b)=b \otimes I+(-1)^{M-\rho+\frac{1}{2}} \otimes b \\
& \Delta\left(b^{\dagger}\right)=b^{\dagger} \otimes I+(-1)^{-M+\rho-\frac{1}{2}} \otimes b^{\dagger} \\
& \varepsilon(K)=-\frac{\delta}{\nu}, \quad \varepsilon(b)=\varepsilon\left(b^{\dagger}\right)=0, \quad \varepsilon(I)=1 \\
& S(K)=K, \quad S(b)=-(-1)^{-M+\rho-\frac{1}{2}} b, \quad S\left(b^{\dagger}\right)=b^{\dagger}(-1)^{M-\rho+\frac{1}{2}} \\
& \Delta\left((-1)^{ \pm M}\right)=(-1)^{ \pm\left(\frac{1}{2}-\rho\right)}(-1)^{ \pm M} \otimes(-1)^{ \pm M}, \\
& S\left((-1)^{ \pm M}\right)=(-1)^{\mp M \pm(2 \rho-1)}, \quad \varepsilon\left((-1)^{ \pm M}\right)=(-1)^{ \pm\left(\rho-\frac{1}{2}\right)}
\end{aligned}
$$


provided that $\nu, \delta \neq 0$. As in the case of $B_{\alpha, \beta}^{+}$it is not necessary to impose at this stage the condition $(-1)^{2 \widetilde{M}}=I$ (which implies that $\left.(-1)^{2 M}=(-1)^{2 \rho-1}\right)$. The form of $\Delta(M), \varepsilon(M)$ and $S(M)$ is given by

$$
\begin{aligned}
& \Delta(M)=M \otimes I+I \otimes M+\left(\frac{1}{2}-\rho\right) I \otimes I, \\
& \varepsilon(M)=\rho-\frac{1}{2}, \quad S(M)=-M+2 \rho-1 .
\end{aligned}
$$

It can be checked that using $\varphi$ and $\varphi^{\prime}$ we can show that the above mentioned isomorphism carries to the Hopf algebra structures of $B_{\alpha, \beta}^{+}$and $H_{\delta, \nu}^{+}$too. An opposite Hopf algebra structure also exists with an antipode the inverse of the one given above:

$$
. S^{-1}(K)=K, \quad S^{-1}(b)=(-1)^{-\widetilde{M}} b, \quad S^{-1}\left(b^{\dagger}\right)=-b^{\dagger}(-1)^{\widetilde{M}}, \quad S^{-1}\left((-1)^{\widetilde{M}}\right)=(-1)^{\widetilde{M}}
$$

Finally we can obtain a realization of $o s p(1 / 2)$ by defining

$$
e=\mu b^{\dagger}, \quad f=\lambda b, \quad h=\frac{\nu}{\delta} K+\frac{2}{\delta} b^{\dagger} b+I
$$

provided that $\mu \lambda=\frac{1}{\delta}$, while for $A_{1}$ (as a subalgebra of $o s p(1 / 2)$ ) by defining

$$
e^{\prime}=\mu^{\prime}\left(b^{\dagger}\right)^{2}, \quad f=\lambda^{\prime} b^{2}, \quad h^{\prime}=\frac{\nu}{2 \delta} K+\frac{1}{\delta} b^{\dagger} b+\frac{1}{2} I
$$

provided $\mu^{\prime} \lambda^{\prime}=-\frac{1}{4 \delta^{2}}$. Implementing the Hopf structure of $H_{\delta, \nu}$ we can obtain a Hopf structure for the bosonization of $\operatorname{osp}(1 / 2)$ as was the case for $B_{\alpha, \beta}^{+}$. In particular it is expected that an $R$ matrix for $H_{\delta, \nu}$ will be of the form of (52) with $\widetilde{M}$ in the place of $\widetilde{N}$ and provided we also demand that $(-1)^{2 \widetilde{M}}=I$.

\section{Conclusion}

In this paper we considered the generalized boson algebras $B_{\alpha, \beta}$ and their $q$-deformed versions $B_{\alpha, \beta}^{q}$ which when enlarged by the element $(-1)^{N}$ it was shown to admit a quasitriangular Hopf algebra structure. In particular this structure revealed the property that $B_{\alpha, \beta}^{+}$and $B_{\alpha, \beta}^{q+}$ can be treated as spectrum generating quantum groups for the undeformed and $q$-deformed bosons respectively. An important point though is the isomorphism of $B_{\alpha, \beta}$ and $H_{\delta, \nu}$ (and their respective enlargements $B_{\alpha, \beta}^{+}$and $H_{\delta, \nu}^{+}$) demonstrated in section 5 which carries over to their Hopf algebra structure. To our knowledge it is the first time that the Calogero-Vasiliev $\nu$-deformed Heisenberg algebra $H_{1, \nu}$, slightly modified (i.e. $K^{2} \neq I$ ), can be formulated as a Hopf algebra. Moreover it is expected that there should exist a $q$-deformation, $H_{\delta, \nu}^{q}$ other than the one of [14] or [13] which may admit a Hopf structure, giving a two-parameter deformation of the Heisenberg algebra and possibly not being isomorphic with $B_{\alpha, \beta}^{q}$, thus giving rise to a new $R$-matrix. Consequences of these Hopf-type boson algebras on physical models such as the Calogero-Sutherland models, supersymmetric quantum mechanics, anyonic systems (whose references mentioned in the introduction) or on radial problems, BRST symmetry [48], are under investigation. It is anticipated that the Hopf algebra structure, and especially the quasitriangular nature of these algebras, might reveal interesting connections with the integrability of the above physical systems. 
Another important aspect of these models is their relations with existing ones. In work under completion, 45], we investigate the various subalgebras of these undeformed and deformed models using the powerful tool of the fixed point set of the adjoint action of a Hopf algebra. It is shown how already known undeformed and $q$-deformed boson algebras appear as fixed point subalgebras or as appropriate quotients. It is at this point that the role of the Cuntz algebra is also investigated. Finally the more natural generalization of the ordinary boson algebra, $\bar{B}_{\sigma, \tau}$, was considered together with its $q$-deformation, and both were shown to be quasitriangular Hopf algebras too.

Braid group representations and possible link invariants for all of the proposed models of deformed and undeformed bosons are worth investigating, while the $\operatorname{ssp}(1 / 2)$ and $A_{1}$ realizations obtained point towards realizations of higher rank algebras and superalgebras which will also allow for the construction of families of infinite dimensional representations when the above Fock-spaces are generalized.

Finally one should comment on the implications of the generalized boson algebras in particular $B_{\alpha, \beta}$, and $B_{\alpha, \beta}^{q}$, for quantum statistics. As the usual oscillator algebra does not possess a Hopf algebra structure, it is difficult to characterize the multiparticle Hamiltonian. However, in our case by generalizing to a many-particle system $B_{\alpha, \beta}^{i} i=1,2, \ldots$ ( and in particular taking $\alpha=2, \beta=1$ which on the Fock space will give $\left.a^{i \dagger} a^{i}=N^{i}, a^{i} a^{i \dagger}=N^{i}+I\right)$ the total Hamiltonian (taken to be proportional to $\alpha \tilde{N}=\left\{a, a^{\dagger}\right\}$ ) has a very natural interpretation as being proportional to $\Delta^{(n)}(\widetilde{N})$, the $n$-fold coproduct of the one particle Hamiltonian. Perhaps of most interest are implications for the quantum statistics of $B_{\alpha, \beta}^{q}$. In this case the existence of the coproduct of $\widetilde{N}$ implies various logical possibilities for the multiparticle Hamiltonian, which may be more acceptable than the obvious (but arbitrary) choice $\propto \sum_{i}\left[N_{i}\right]_{q}$ which has no justification in terms of a Hopf structure. Non-local effects will likely emerge from such choices, which may play a crucial role in modifying the partition functions and statistics of the system.

\section{Acknowledgments}

The authors would like to thank P. E. T. Jørgensen, A. J. Bracken, D. S. McAnally and R. Zhang for their sincere interest, support and fruitful comments during the completion of this work parts of which were reported in the 12th Australian Institute of Physics Congress, Hobart July 1-5, 1996 and in the 3rd International Conference on Functional Analysis and Approximation Theory, September 23-28, 1996 Acquafredda di Maratea, Potenza, Italy [49].

\section{References}

[1] Drinfeld V G 1986 "Quantum Groups" in Proc. ICM Berkeley, 1 798;

[2] Jimbo M. 1985 Lett. Math. Phys. 10 63; 1986 Lett. Math. Phys. 11 247; 1987 Commun. Math. Phys. 102 537; "Quantum Groups" 1990 Proceedings of the Argonne Workshop World Scientific 1990 ed. Curtright T, Fairlie D and Zachos C.

[3] Woronowicz S L 1987 Comm. Math. Phys. 111 613; Woronowicz S L 1987 Publ. RIMS 23, 117-181; Woronowicz S L 1988 Inventiones Math. 93 35;

[4] Links R J and Gould M D 1992 Rep. Math. Phys. 31 91; Gould M D, Zhang R B and Bracken A J 1991 J. Math. Phys. 322298

[5] Vyjayanthi Chari and Andrew Pressley 1995 'A Guide to Quantum Groups' Cambridge Univ. Press, Melbourne; 
[6] Gould M D, Zhang R B and Bracken A J 1993 Bull. Austral. Math. Soc. 47 353; Khoroshkin S M and Tolstoy V N 1991 Comm. Math. Phys. 141 599; Kulish P P Reshetikhin N Y 1989 Lett. Math. Phys.18 143; Bracken A J, Gould M D and Tsohantjis I 1993 J. Math. Phys. 34 1654; Bracken A J, Gould M D and Zhang R B 1990 Mod. Phys. Lett. A 5 831; Links R J, Scheunert M and Gould M D 1994 Lett. Math. Phys. 32 231; Zhang R B 1993 J. Math. Phys. 34 1236; Zhang R B 1993 J. Phys. A: Math. Gen 26 7041;

[7] Arik M and Coon D D 1976 J. Math. Phys.. 17 524;

[8] Macfarlane A J 1989 J. Phys. A: Math. and Gen. 22 4581;

[9] Biedenharn L C 1989 J. Phys. A: Math. Gen. 22 L873;

[10] Sun C-P and Fu H-C 1989 J. Phys. A: Math. Gen. 22 L983;

[11] Chakrabarti R and Jaganathan R 1991 J. Phys. A: Math. Gen. 24 L711;

[12] Vasiliev M A 1989 Pis'ma JETP 50 344; 1991 Inter. J. Mod. Phys. A6 1115;

[13] Brzezinski T, Egusquiza I L and Macfarlane A J 1993 Phys. Lett. 311B 202;

[14] Macfarlane A J 1994 J. Math. Phys. 35 1054;

[15] Kuryshkin W 1980 Ann. Found. L de Broglie 5 111;

[16] Jannussis A et al 1982 Hadronic J. 5 1923; Jannussis A, Brodimas G, Sourlas D and Zisis, V 1981 Lett. Nuovo Cimento 30 123; Jannussis A 1991 Hadronic J. 14 257; Brodimas G "Lie Admissible Q-Algebras and Quantum Groups" Phd Thesis 1991 University of Patras, Patras, Greece;

[17] Ng Y T 1990 J. Phys. A: Math. and Gen. 23 1023;

[18] Kulish P P and Damaskinsky E V 1990 J. Phys. A: Math. Gen. 23 L415;

[19] Chaichian M and Kulish P P 1990 Phys. Lett. 234B 72;

[20] Chaichian M and Ellinas D 1990 J. Phys. A: Math. and Gen. 23 L291;

[21] Chaichian M, Kulish P P and Lukierski J 1990 Phys. Lett. 237B 401;

[22] Sun Chang-Pu and Ge Mo-Lin 1991 J. Math. Phys. 32 595;

[23] Oh C H and Singh K 1994 J. Phys. A: Math. Gen. 27 5907;

[24] Cho K-H and Park S U 1995 J. Phys. A: Math. Gen. 28 1005;

[25] Daskaloyannis C 1991 J. Phys. A: Math. Gen. 24 L789;

[26] Bracken A J, McAnally D S, Zhang R B and Gould M D 1991 J. Phys. A: Math. Gen .24 1379 ;

[27] McDermott R J and Solomon 1994 J. Phys. A: Math. Gen. 24 L15;

[28] Katriel J and Quesne C 1996 J. Math. Phys. 37 1650;

[29] Plyushchay M S 1994 Phys. Lett. 320B 91; 1996 Ann. Phys. 245 339; 
[30] Plyushchay M S 1996 Ann. Phys. 245 339;

[31] Brink L, Hansson T H and Vasiliev M A 1992 Phys. Lett. 286B 109; Brink L, Hansson T H, Konstein S and Vasiliev M A 1993 Nucl. Phys. 401B 591;

[32] Calogero F 1969 J. Math. Phys. 10 2191; 1971 J. Math. Phys. 12 419; Sutherland B 1971 J. Math. Phys. 12 246;

[33] Calogero F 1995 Phys. Lett. 201A 306; 1995 J. Math. Phys. 36 9; Calogero F and van Diejen J F 1995 Phys. Lett. 205A 143;

[34] Jørgensen P E T, Schmitt L M and Werner R F 1994 Pacific. J. Math. 165 131;

[35] Jørgensen P E T, Schmitt L M and Werner R F 1995 J. Fun. Anal. 134 33;

[36] Jørgensen P E T, Schmitt L M and Werner R F 1994 ' $q$-relations and stability of C*isomorphism classes' in Algebraic Methods in Operator Theory ed. Curto R and Jørgensen P E T 1994 Birkhäuser Boston;

[37] Jørgensen P E T 1994 Contemporary Math. 160 141;

[38] Jørgensen P E T and Werner R F 1994 Commun. Math. Phys. 164 455;

[39] Chaichian M, Grosse H and Presnajder 1994 J. Phys. A: Math. Gen. 27 2045; Chaichian M, Gonzalez Felipe R and Presnajder 1995 J. Phys. A: Math. Gen. 28 2247;

[40] Palev T D "Is it possible to extend the deformed Weyl algebra $W_{q}(n)$ to a Hopf algebra?" ICTP, Trieste IC-93-163, hep-th/9307032;

[41] Hong Yan 1990 J. Phys. A: Math. Gen. 23 L1155;

[42] Jian-Hui Dai, Han-Ying Guo and Hong Yan 1991 J. Phys. A: Math. Gen. 24 L409;

[43] Hong Yan 1991 Phys. Lett. 262B 459;

[44] McAnally D S and Tsohantjis I 'Deformed boson algebras and the quantum double construction' to appear in J. Phys. A: Math. Gen. .

[45] Paolucci A and Tsohantjis I 'On Hopf-type boson algebras and their fixed point subalgebras' in preparation.

[46] Macfarlane A J and Majid S 1992 Int. J. Mod. Phys. 7A 4377

[47] Sweedler M E 1969 Hopf Algebras (Benjamin, NY)

[48] Bracken A J and Leemon H I 1980 J. Math. Phys. 21 2170; 1981 J. Math. Phys. 22 719; Jarvis P D and Baker T H 1992 J. Phys. A:Math. Gen. 26 883; Jarvis P D, Warner R C, Yung C M and Zhang R B 1992 J Phys A25 L895-L900;

[49] Tsohantjis I, Jarvis P D and Paolucci A 'Oscillator algebras as Hopf algebras' poster presented at the 12th Australian Institute of Physics Congress, Hobart July 1-5, 1996; Paolucci A, Tsohantjis I and Jarvis P D 'A new example of boson algebras and generalized harmonic oscillators' talk presented at the 3rd International Conference on Functional Analysis and Approximation Theory, September 23-28, 1996 Acquafredda di Maratea, Potenza Italy, to appear in the proceedings. 